Eastern Illinois University

The Keep

Faculty Research \& Creative Activity

Biological Sciences

October 2007

\title{
Impact of carbon sources on growth and oxalate synthesis by the phytopathogenic fungus Sclerotinia sclerotiorum
}

\author{
Steven L. Daniel \\ Eastern Illinois University, sldaniel@eiu.edu \\ Bryan J. Culbertson \\ Eastern Illinois University \\ Jaymie Krone \\ Eastern Illinois University \\ Norbert Furumo \\ University of Hawaii at Hilo
}

Follow this and additional works at: http://thekeep.eiu.edu/bio_fac

Part of the Bacteriology Commons, Environmental Microbiology and Microbial Ecology Commons, and the Microbial Physiology Commons

\section{Recommended Citation}

Daniel, Steven L.; Culbertson, Bryan J.; Krone, Jaymie; and Furumo, Norbert, "Impact of carbon sources on growth and oxalate synthesis by the phytopathogenic fungus Sclerotinia sclerotiorum" (2007). Faculty Research \& Creative Activity. 122.

http://thekeep.eiu.edu/bio_fac/122 


\title{
Impact of carbon sources on growth and oxalate synthesis by the phytopathogenic fungus Sclerotinia sclerotiorum
}

\author{
Bryan J. Culbertson, Jaymie Krone, Erastus Gatebe, Norbert C. Furumo, and
}

Steven L. Daniel

World Journal of Microbiology and Biotechnology, October 2007, Volume 23, Issue 10, pp 1357-1362

\begin{abstract}
The impact of various supplemental carbon sources (oxalate, glyoxylate, glycolate, pyruvate, formate, malate, acetate, and succinate) on growth and oxalate formation (i.e., oxalogenesis) by Sclerotinia sclerotiorum was studied. With isolates D-E7, 105, W-B10, and Arg-L of S. sclerotiorum, growth in an undefined broth medium $0.1 \%$ soytone; pH 5) with $25 \mathrm{mM}$ glucose and $25 \mathrm{mM}$ supplemental carbon source was increased by the addition of malate and succinate. Oxalate accumulation occurred in the presence of glucose and a supplemental carbon source, with malate, acetate, and succinate supporting the most oxalate synthesis. With S. sclerotiorum Arg-L, oxalate-to-biomass ratios, an indicator of oxalogenic potential, were dissimilar when the organism was grown in the presence of different carbon sources. The highest oxalate-to-biomass ratios were observed with pyruvate, formate, malate, acetate, and succinate. Time-course studies with acetate-supplemented cultures revealed that acetate and glucose consumption by S. sclerotiorum D-E7 coincided with oxalogenesis and culture acidification. By day 5 of incubation, oxalogenesis was halted when cultures reached a $\mathrm{pH}$ of 3 and were devoid of acetate. In succinatesupplemented cultures, oxalogenesis essentially paralleled glucose and succinate utilization over the 9-day incubation period; during this time period, culture $\mathrm{pH}$ declined but never fell below 4. Overall, these results indicate that carbon sources can regulate the accumulation of oxalate, a key pathogenicity determinant for $\mathrm{S}$. sclerotiorum.
\end{abstract}

\section{Introduction}

Sclerotinia sclerotiorum is a phytopathogenic fungus that significantly reduces the yields and quality of agricultural crops worldwide each year (Purdy 1979; Willetts and Wong 1980; Wrather et al. 1997; Bolton et al. 2006). In addition, this ubiquitous ascomycete has a wide host range and can infect over 400 species of plants (Purdy 1979; Willetts and Wong 1980; Steadman 1983; Boland and Hall 1994; Grau and Hartman 1999). For S. sclerotiorum, oxalate (-OOC-COO-) production is a key pathogenicity determinant (Maxwell and Lumsden 1970; Noyes and Hancock 1981; Marciano et al. 1983; Magro et al. 1984; Godoy et al. 1990; Durman et al. 2005; Guimarães and Stolz 2005; Hegedus and Rimmer 2005; Bolton et al. 2006). While the role and importance of S. sclerotiorum-derived oxalate in plant disease has been 
extensively studied, information on the actual mechanism and regulation of oxalate synthesis by S. sclerotiorum is quite limited.

In S. sclerotiorum, oxalate formation (i.e., oxalogenesis) appears to be due to oxaloacetate acetylhydrolase (EC 3.7.1.1) which catalyses the conversion of oxaloacetate to acetate and oxalate (Maxwell 1973). Simple and complex carbohydrates (glucose, mannitol, sucrose, pectin, polygalacturonic acid, carboxymethylcellulose, xylan, and onion- and sunflower-derived cell wall components) are utilized by S. sclerotiorum and converted to oxalate (Maxwell and Lumsden 1970; Vega et al. 1970; Marciano et al. 1989; Rollins and Dickman 2001). Furthermore, during glucose-dependent growth and oxalogenesis by S. sclerotiorum, the addition of succinate to culture media causes an increase in oxalate accumulation (Maxwell and Lumsden 1970; Godoy et al. 1990). How succinate, as well as other carbon sources, regulates oxalogenesis by S. sclerotiorum remains to be resolved. Therefore, the goals of the present study were to examine the impact of different carbon sources (organic acids) on growth and oxalate synthesis by $\mathrm{S}$. sclerotiorum.

\section{Materials and methods}

\section{Source of S. sclerotiorum isolates}

Sclerotinia sclerotiorum D-E7, 105, W-B10, and Arg-L were obtained from the National Soybean Pathogen Collection Center at the University of Illinois at UrbanaChampaign. D-E7, 105, W-B10, and Arg-L were originally isolated from infected soybeans in Illinois, Iowa, Illinois, and Argentina, respectively.

\section{Growth of S. sclerotiorum in culture media}

Sclerotinia sclerotiorum was grown in 125-ml culture flasks containing $50 \mathrm{ml}$ of undefined medium. The undefined medium contained (mg/l): 1,000 soytone (Difco); $\left(\mathrm{NH}_{4}\right)_{2} \mathrm{SO}_{4}, 1,000 ; \mathrm{K}_{2} \mathrm{HPO}_{4}, 500 ; \mathrm{KH}_{2} \mathrm{PO}_{4}, 500 ; \mathrm{NaCl}, 450 ; \mathrm{MgSO}_{4} \cdot 7 \mathrm{H}_{2} 0,250$; Na.nitrilotriacetate, 5; $\mathrm{FeCl}_{3} \cdot 6 \mathrm{H}_{2} 0,0.5 ; \mathrm{CuSO}_{4} \cdot 5 \mathrm{H}_{2} \mathrm{O}, 0.5 ; \mathrm{ZnCl}_{2}, 0.5 ; \mathrm{MnSO}_{4} \cdot \mathrm{H}_{2} \mathrm{O}$; and $\mathrm{Na}_{2} \mathrm{MoO}_{4} \cdot 2 \mathrm{H}_{2} \mathrm{O}, 0.5$. The $\mathrm{pH}$ of the culture medium was adjusted to 5 and sterilized by autoclaving. d-glucose and supplemental carbon sources (oxalate, glyoxylate, glycolate, pyruvate, formate, malate, acetate, and succinate) were prepared in deionized water (when necessary, free acids were neutralized with $6 \mathrm{M} \mathrm{KOH}$ ) and filter-sterilized. Prior to inoculation, glucose and supplemental carbon sources were added from filter-sterilized stock solutions to flasks of sterile culture medium to the initial concentrations indicated.

Isolates of S. sclerotiorum were maintained on Petri plates of undefined medium (pH 5) containing $25 \mathrm{mM}$ glucose and solidified with $1.5 \%$ agar. Inoculation was accomplished by removing a 5 -mm plug (cut with a sterile cork borer) of mycelium from the advancing edge of growth and placing the plug, mycelium side down, centrally on the surface of the sterile agar medium. Inoculated plates were placed in 
plastic zip-lock bags (partially sealed) and incubated at $25^{\circ} \mathrm{C}$ in the dark. Growth in flasks was initiated with a single 5-mm agar-mycelial plug of S. sclerotiorum cut from the advancing edge of an agar culture. After inoculation, culture flasks were incubated at $25^{\circ} \mathrm{C}$ with shaking at $180 \mathrm{rev} / \mathrm{min}$ for 9 days (unless indicated otherwise). After the designated incubation period, the following parameters were measured in each culture: growth (biomass formed); the concentration of oxalate, supplemental carbon source, and glucose; and culture $\mathrm{pH}$.

\section{Analytical methods}

For biomass determinations, mycelium from each culture flask was collected by vacuum filtration through a Büchner funnel containing a pre-weighed Whatman No. 1 filter paper; samples of culture filtrates were saved for analysis by highperformance liquid chromatography and for $\mathrm{pH}$ determination. Collected fungal biomass was oven-dried at $55^{\circ} \mathrm{C}$ for 3 days, cooled to room temperature in a desiccator, and then weighed. Growth was expressed as biomass formed [mg (dry weight) per flask].

Culture filtrates were re-filtered using a 25-mm syringe filter (nylon; pore size, $0.2 \mu \mathrm{m}$; Fisher Scientific) and the concentrations of oxalate, glucose, and supplemental carbon sources were determined using a Beckman Gold highperformance liquid chromatograph fitted with a 300-mm Bio-Rad Aminex HPX-87H column. Chromatographic conditions included: column temperature, $55^{\circ} \mathrm{C}$; mobile phase, $0.01 \mathrm{~N} \mathrm{H}_{2} \mathrm{SO}_{4}$; flow rate of mobile phase, $0.6 \mathrm{ml} / \mathrm{min}$; and injection volume, $10 \mu \mathrm{l}$. Detection of oxalate and supplemental carbon sources was done at $210 \mathrm{~nm}$, and glucose detection was done with a refractive index detector. Oxalate, supplemental carbon source, and glucose concentrations were expressed on a millimolar basis. The $\mathrm{pH}$ of culture filtrates was determined with an Orion model $230 \mathrm{~A} \mathrm{pH}$ meter and an Orion semi-micro combination electrode. In this study, no distinctions were made between organic acids and their salt forms.

\section{Results and discussion}

Isolates (D-E7, 105, W-B10, and Arg-L) of S. sclerotiorum were examined for their ability to grow and synthesize oxalate in the presence of glucose and a supplemental carbon source (Table 1). Relative to growth, of the eight different carbon sources examined (oxalate, glyoxylate, glycolate, pyruvate, formate, malate, acetate, and succinate), only malate and succinate clearly stimulated glucose-dependent growth of all four S. sclerotiorum isolates (Table 1). The growth of isolates D-E7 and W-B10 was also stimulated by pyruvate. Other carbon sources appeared to be stimulatory but the range of growth often observed for a given isolate was large, thus making comparisons within isolates difficult. In addition, there was considerable variation in the amount of growth that occurred between isolates. Isolates 105 and W-B10 displayed marginal growth (regardless of the presence or absence of a supplemental carbon source) after 9 days of incubation (Table 1). This was not the case with isolates with D-E7 and W-B10. Differences in growth potentials are known to occur 
among isolates of S. sclerotiorum, including isolates grown in succinate-containing broth cultures (Maxwell and Lumsden 1970; Marciano et al. 1989).

\begin{tabular}{|c|c|c|c|c|c|c|c|c|}
\hline \multirow{2}{*}{$\begin{array}{l}\text { Supplemental } \\
\text { carbon } \\
\text { source }^{\mathrm{a}}\end{array}$} & \multicolumn{4}{|c|}{ Biomass formed [mg (dry weight) per flask] } & \multicolumn{4}{|c|}{ Oxalate formed (mM) } \\
\hline & D-E7 & 105 & W-B10 & Arg-L & D-E7 & 105 & W-B10 & Arg-L \\
\hline None (control) & $122 \pm 14$ & $12 \pm 3$ & $19 \pm 5$ & $85 \pm 2$ & $0 \pm 0$ & $2.0 \pm 0.0$ & $1.7 \pm 0.1$ & $0 \pm 0$ \\
\hline Oxalate & $172 \pm 48$ & $13 \pm 3$ & $17 \pm 11$ & $86 \pm 10$ & $3.0 \pm 0.4$ & $1.9 \pm 0.2$ & $0.9 \pm 0.2$ & $0.9 \pm 0.6$ \\
\hline Glyoxylate & $116 \pm 32$ & $8 \pm 3$ & $12 \pm 3$ & $99 \pm 11$ & $7.0 \pm 0.3$ & $4.5 \pm 0.1$ & $4.0 \pm 0.2$ & $5.4 \pm 0.2$ \\
\hline Glycolate & $159 \pm 40$ & $10 \pm 1$ & $16 \pm 2$ & $106 \pm 18$ & $9.0 \pm 0.7$ & $5.6 \pm 0.2$ & $2.3 \pm 0.1$ & $7.5 \pm 0.2$ \\
\hline Pyruvate & $198 \pm 14$ & $17 \pm 3$ & $32 \pm 2$ & $98 \pm 7$ & $12.8 \pm 0.5$ & $6.0 \pm 0.1$ & $5.3 \pm 0.1$ & $12.1 \pm 0.2$ \\
\hline Formate & $134 \pm 9$ & $10 \pm 4$ & $18 \pm 0$ & $99 \pm 10$ & $15.7 \pm 0.3$ & $7.2 \pm 0.1$ & $3.9 \pm 0.1$ & $14.8 \pm 0.2$ \\
\hline Malate & $258 \pm 13$ & $30 \pm 2$ & $50 \pm 1$ & $113 \pm 4$ & $18.9 \pm 0.3$ & $11.2 \pm 0.3$ & $11.6 \pm 0.7$ & $18.5 \pm 0$ \\
\hline Acetate & $102 \pm 32$ & $18 \pm 4$ & $28 \pm 2$ & $78 \pm 13$ & $15.2 \pm 1.1$ & $16.0 \pm 0.5$ & $15.1 \pm 0.4$ & $14.1 \pm 0.9$ \\
\hline Succinate & $192 \pm 38$ & $30 \pm 1$ & $41 \pm 4$ & $130 \pm 3$ & $26.6 \pm 0.7$ & $22.2 \pm 0.3$ & $16.9 \pm 1.1$ & $25.4 \pm 0.2$ \\
\hline
\end{tabular}

Each value represents the mean of duplicate or triplicate cultures \pm the standard deviation. Measurements were made after 9 days of incubation at $25^{\circ} \mathrm{C}$ with shaking aSupplemental carbon sources $(25 \mathrm{mM})$ were added to the undefined medium containing $25 \mathrm{mM}$ glucose

\section{Table 1}

Effect of supplemental carbon sources on growth and oxalate formation by different isolates (D-E7, 105, W-B10, and Arg-L) of Sclerotinia sclerotiorum

In contrast, oxalate accumulation occurred with all isolates in the presence of a supplemental carbon source, and, in most cases, was increased (Table 1). Among the carbon sources examined, malate, acetate, and succinate were the best in promoting oxalate synthesis by these four isolates of S. sclerotiorum. With malate or succinate present, even low-growth-yielding isolates 105 and W-B10 yielded elevated oxalate levels that approached those in cultures of high-growth-yielding isolates D-E7 and Arg-L. Moreover, in acetate-containing cultures, oxalate concentrations were essentially identical for all four isolates (Table 1). Thus, there was no connection between oxalate accumulation and growth under these culture conditions. Our results support previous findings that oxalate production by S. sclerotiorum and other oxalate-producing phytopathogenic fungi is not always correlated with biomass formation (Maxwell and Bateman 1968a; Maxwell and Lumsden 1970; 
Pierson and Rhodes 1992; Briere et al. 2000). To provide insight into the relationship between supplemental carbon source, growth, and oxalogenesis, further studies were conducted with S. sclerotiorum Arg-L and D-E7. With S. sclerotiorum Arg-L, the impact of supplemental carbon sources on substrate-product profiles, oxalate-to-biomass ratios, and culture $\mathrm{pH}$ was examined (Table 2). Glucose and supplemental carbon sources (with the exception of oxalate, glyoxylate, and glycolate) were for the most part totally consumed by the end of the 9-day incubation period. The oxalate-to-biomass ratio is often used as an indicator of the oxalogenic potential of S. sclerotiorum during growth (Durman et al. 2005). In this study, oxalate-to-biomass ratios were dissimilar for Arg-L, indicating the organism's ability to grow and accumulate oxalate in the presence of these different carbon sources was not equivalent (Table 2). The highest oxalate-to-biomass ratios were observed in the presence of pyruvate, formate, malate, acetate, or succinate. How these various carbon sources, especially formate (a one-carbon compound), are metabolized by S. sclerotiorum and how this metabolism leads to the increased accumulation of oxalate have yet to be determined.

\begin{tabular}{|c|c|c|c|c|c|c|}
\hline $\begin{array}{l}\text { Supplemental } \\
\text { carbon } \\
\text { source }^{\mathrm{a}}\end{array}$ & $\begin{array}{l}\text { Biomass } \\
\text { formed [mg } \\
\text { (dry } \\
\text { weight)/flask] }\end{array}$ & $\begin{array}{l}\text { Supplemental } \\
\text { carbon } \\
\text { source } \\
\text { consumed } \\
(\mathrm{mM})\end{array}$ & $\begin{array}{l}\text { Oxalate } \\
\text { formed } \\
(\mathrm{mM})\end{array}$ & $\begin{array}{l}\text { Oxalate- } \\
\text { to-biomass } \\
\text { ratioc }\end{array}$ & $\begin{array}{l}\text { Final } \\
\text { culture } \\
\mathrm{pH}\end{array}$ & $\underset{\mathrm{a}}{\mathrm{pK}}$ \\
\hline None (control) & $85 \pm 2$ & $N A^{b}$ & $0 \pm 0$ & 0 & $3.5 \pm 0.1$ & NA \\
\hline Oxalate & $86 \pm 10$ & $0 \pm 0$ & $0.9 \pm 0.6$ & 0.5 & $4.5 \pm 0.1$ & $\begin{array}{l}1.23 \\
4.19\end{array}$ \\
\hline Glyoxylate & $99 \pm 11$ & $7.1 \pm 0.6$ & $5.4 \pm 0.2$ & 2.7 & $4.4 \pm 0.1$ & 3.18 \\
\hline Glycolate & $106 \pm 18$ & $8.4 \pm 0.7$ & $7.5 \pm 0.2$ & 3.5 & $4.0 \pm 0.1$ & 3.83 \\
\hline Pyruvate & $98 \pm 7$ & $25 \pm 0$ & $12.1 \pm 0.2$ & 6.2 & $4.5 \pm 0.1$ & 2.39 \\
\hline Formate & $99 \pm 10$ & $22.1 \pm 0.7$ & $14.8 \pm 0.2$ & 7.5 & $4.2 \pm 0.1$ & 3.75 \\
\hline Malate & $113 \pm 4$ & $25 \pm 0$ & $18.5 \pm 0$ & 8.2 & $5.3 \pm 0.1$ & $\begin{array}{l}3.40 \\
5.11\end{array}$ \\
\hline Acetate & $78 \pm 13$ & $24.7 \pm 0.2$ & $14.1 \pm 0.9$ & 9.0 & $4.3 \pm 0.1$ & 4.76 \\
\hline Succinate & $130 \pm 3$ & $24.8 \pm 0.1$ & $25.4 \pm 0.2$ & 9.8 & $4.4 \pm 0.1$ & $\begin{array}{l}4.16, \\
5.61\end{array}$ \\
\hline
\end{tabular}


Each value represents the mean of triplicate cultures \pm the standard deviation. Measurements were made after 9 days of incubation at $25^{\circ} \mathrm{C}$ with shaking

aSupplemental carbon sources were added to the undefined medium containing $25 \mathrm{mM}$ glucose. In all cultures, glucose was totally consumed by day 9 of incubation b NA not applicable. Cultures were grown in the undefined medium with glucose only (no supplemental carbon source)

cPer flask (50-ml culture): micromoles of oxalate formed per unit (milligram) of biomass formed

${ }^{\mathrm{d}} \mathrm{pK} \mathrm{K}_{\mathrm{a}}\left(25^{\circ} \mathrm{C}\right)$ of each supplemental carbon source (organic acid) used in this study (ZirChrom 2006)

\section{Table 2}

Effect of supplemental carbon sources on substrate-product profiles, oxalate-tobiomass ratios, and culture $\mathrm{pH}$ of Sclerotinia sclerotiorum Arg-L

It is tempting to speculate that as tricarboxylic acid (TCA) cycle intermediates, malate and succinate are direct sources of oxaloacetate, another TCA-level intermediate which is apparently hydrolyzed by S. sclerotiorum to oxalate (Maxwell 1973). Likewise, pyruvate carboxylase, a gluconeogenic enzyme which converts pyruvate to oxaloacetate, may account for pyruvate consumption, and hence, the formation of oxalate (Table 2). Also, pyruvate dehydrogenase converts pyruvate to acetyl-CoA which may then enter the TCA cycle; however, no net accumulation of oxaloacetate can occur via this route due to the release of two molecules of $\mathrm{CO}_{2}$ for every acetyl-CoA entering the cycle. Perhaps a more plausible explanation for the consumption of acetyl-CoA (from acetate or pyruvate) is via the glyoxylate pathway which bypasses the $\mathrm{CO}_{2}$-generating steps of the TCA cycle. This pathway is essential in plants and in some bacteria, algae, and fungi for glucose synthesis (Lorenz and Fink 2001). Furthermore, acetyl-CoA is an allosteric activator of pyruvate carboxylase leading to a net increase in oxaloacetate from pyruvate, an anaplerotic reaction of the TCA cycle. Unfortunately, not enough is known about pyruvate carboxylase in S. sclerotiorum to say whether it is regulated in this manner. While oxalate yields and oxalate-to-biomass ratios were improved (compared to controls) when glyoxylate or glycolate were present, the increases observed were much less than those observed for acetate or succinate (Table 2). These results suggest that glyoxylate and glycolate were not readily metabolized to oxalate by S. sclerotiorum. With some fungal phytopathogens (e.g., Sclerotium rolfsii), glyoxylate is converted to oxalate via glyoxylate dehydrogenase (Maxwell and Bateman 1968b; Dutton and Evans 1996; Gadd 1999).

In addition to carbon source, culture $\mathrm{pH}$ is also known to regulate oxalate accumulation by S. sclerotiorum, and oxalate formation is increased when the $\mathrm{pH}$ or buffering capacity of the medium is increased (Maxwell and Lumsden 1970; Rollins and Dickman 2001; Bolton et al. 2006). Indeed, S. sclerotiorum does not accumulate oxalate when the $\mathrm{pH}$ of the culture medium is equal to or less than 3.5 (Maxwell and Lumsden 1970). This may explain why control cultures of Arg-L, with a final culture 
$\mathrm{pH}$ of 3.5, failed to form oxalate (Table 2). This also suggests that the supplemental carbon sources used in this study, besides serving as potential oxalogenic substrates, may also serve to increase oxalate production by enhancing the buffering capacity of the medium, a minimally buffered medium with approximately $6.5 \mathrm{mM}$ phosphate buffer. For most supplemented cultures (Table 2), final $\mathrm{pH}$ values were similar ( $\mathrm{pH} 4-4.5)$ and were not related to the amount of oxalate formed or the $\mathrm{pK}_{\mathrm{a}}$ of the carbon source (organic acid) added to the medium. Nonetheless, it is interesting to note that the highest oxalate yields were in cultures supplemented with malate and succinate, organic acids with the highest $\mathrm{pK}_{\mathrm{a}}$ values of those tested (Table 2).

The relationship between supplemental carbon sources (acetate or succinate), oxalogenesis, and culture $\mathrm{pH}$ during growth was examined with S. sclerotiorum DE7 (Fig. 1). In control cultures (no supplemental carbon source), growth coincided with glucose consumption and rapid acidification of the medium; culture $\mathrm{pH}$ remained at this low level $(\mathrm{pH}<3.5)$ during the rest of the 9-day incubation period (Fig. 1a). During this time, oxalate levels were minimal $(<1 \mathrm{mM})$ indicating that acidification prevented oxalate accumulation from the onset of growth. In acetatesupplemented cultures, growth and glucose consumption occurred concurrently up to day 7 whereas oxalate accumulation ceased after day 5 (Fig. 1b). This stoppage coincided with the total consumption of acetate and a drop in culture $\mathrm{pH}$ to less than 3.5. Whether oxalogenesis was halted by acidification (e.g., due to the loss in buffering capacity since acetate was rapidly consumed), by the absence of acetate as an oxalogenic precursor, or by a combination of these two is presently unclear. What is also unclear is why culture $\mathrm{pH}$ increased after day 5 , reaching a value of approximately 4.5 on day 9 . One possibility may involve an intracellular oxalate decarboxylase (EC 4.1.1.2) in S. sclerotiorum, which catalyses the following reaction: $-\mathrm{OOC}-\mathrm{COO}^{-}+\mathrm{H}^{+} \rightarrow \mathrm{HCOO}^{-}+\mathrm{CO}_{2}$ (Magro et al. 1988). This proton-consuming reaction could account for the increasing $\mathrm{pH}$ and decreasing oxalate levels observed in cultures after day 5 (Fig. 1b). In succinate-supplemented cultures (Fig. 1c), a slightly different pattern was observed. Growth and oxalate yields increased over the 9-day incubation period and were essentially concomitant with glucose and succinate consumption. Culture $\mathrm{pH}$, on the other hand, decreased during growth but never dropped below a value of 4 even as succinate levels decreased (Fig. 1c). 

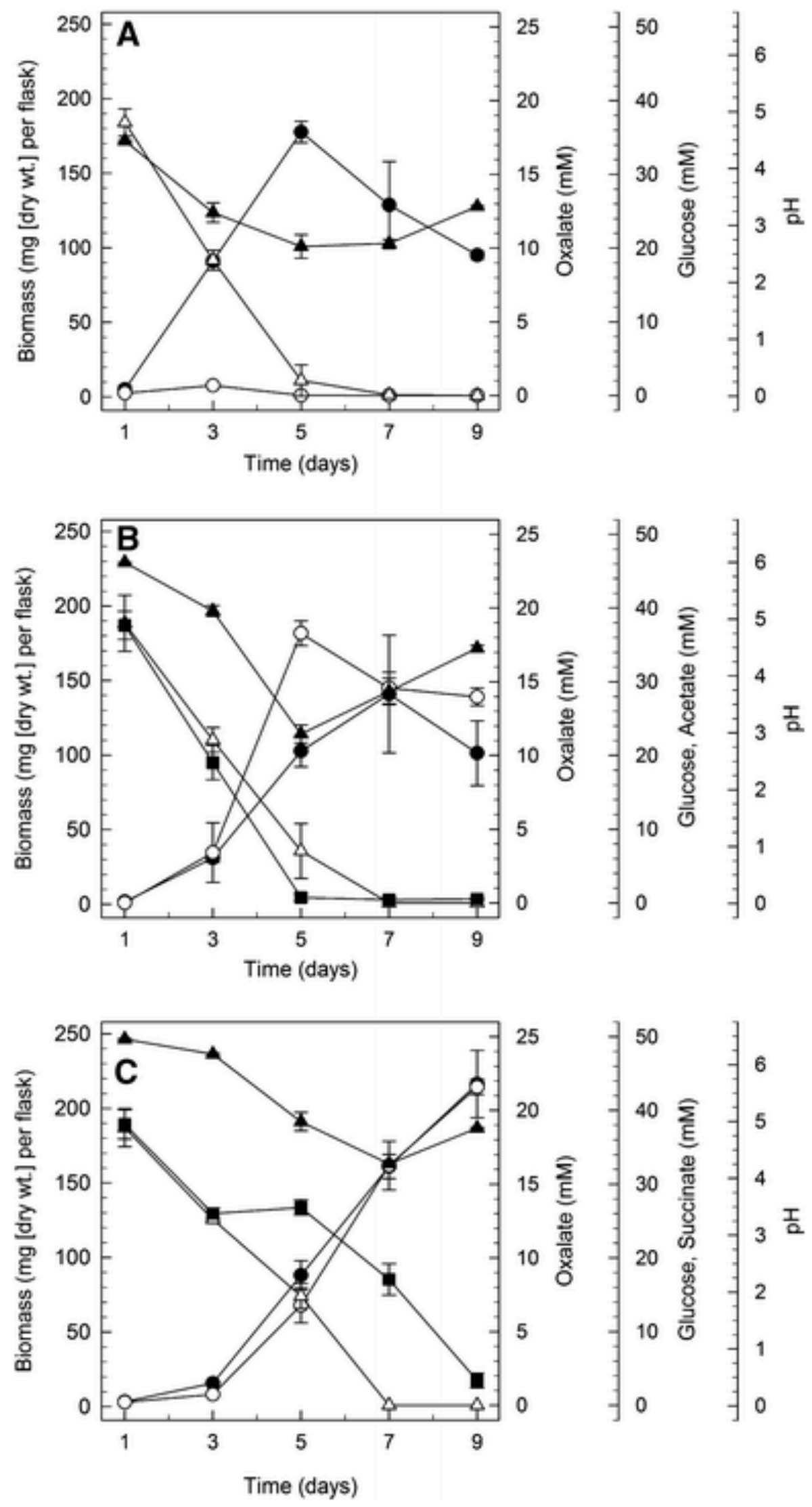


\section{Fig. 1}

Time-course analysis of oxalate formation, glucose and supplemental carbon source consumption, and culture $\mathrm{pH}$ for Sclerotinia sclerotiorum D-E7 during growth at $25^{\circ} \mathrm{C}$ (with shaking) in the undefined medium containing glucose (a); glucose and acetate (b); and glucose and succinate (c). The concentrations of glucose and supplemental carbon sources (acetate or succinate) approximated $40 \mathrm{mM}$. Symbols: biomass, $(\bullet)$; glucose, $(\Delta)$; acetate or succinate, $(\boldsymbol{\bullet})$, oxalate, $(\mathrm{O})$; and $\mathrm{pH},(\boldsymbol{\Delta})$. Each value represents the mean \pm standard deviation of measurements from triplicate cultures

\section{Conclusion}

Sclerotinia sclerotiorum is an important phytopathogenic fungus that is highly dependent upon the production of oxalate, a toxin, for plant infection and disease (Hegedus and Rimmer 2005; Bolton et al. 2006). Strains of S. sclerotiorum which yield higher oxalate levels exhibit more pathogenicity than low oxalate-yielding strains (Maxwell and Lumsden 1970; Marciano et al. 1983; Godoy et al. 1990; Ziman et al. 1998). Thus, understanding the mechanism of oxalate synthesis and secretion by S. sclerotiorum and how this process is regulated may provide us with the strategies necessary to control this global plant pathogen. In the present study, we have demonstrated that carbon sources have the potential to regulate oxalate accumulation by S. sclerotiorum; however, elucidation of the exact nature of this regulatory process will require further investigation.

\section{Acknowledgments}

Support for this study was provided by the Council on Faculty Research at Eastern Illinois University and by a grant from the Illinois Soybean Program Operating Board.

\section{References}

Boland GJ, Hall R (1994) Index of plant hosts of Sclerotinia sclerotiorum. Can J Plant Pathol 16:93-108

Bolton MD, Thomma BPHJ, Nelson BD (2006) Sclerotinia sclerotiorum (Lib.) de Bary: biology and molecular traits of a cosmopolitan pathogen. Mol Plant Pathol $7: 1-16$

Briere SC, Watson AK, Hallett SG (2000) Oxalic acid production and mycelial biomass yield of Sclerotinia minor for the formulation enhancement of a granular turf bioherbicide. Biocontrol Sci Technol 10:281-289

Durman SB, Menendez AB, Godeas AM (2005) Variation in oxalic acid production and mycelial compatibility within field populations of Sclerotinia sclerotiorum. Soil Biol Biochem 37:2180-2184 
Dutton MV, Evans CS (1996) Oxalate production by fungi: its role in pathogenicity and ecology in the soil environment. Can J Microbiol 42:881-895

Gadd GM (1999) Fungal production of citric and oxalic acid: importance in metal speciation, physiology and biochemical processes. Adv Microb Physiol 41:47-92

Godoy G, Steadman JR, Dickman MB, Dam R (1990) Use of mutants to demonstrate the role of oxalic acid in pathogenicity of Sclerorinia sclerotiorum on Phaseolus vulgaris. Physiol Mol Plant Pathol 37:179-191

Grau CR, Hartman GL (1999) Sclerotinia stem rot. In: Hartman GL, Sinclair JB, Rupe JC (eds) Compendium of soybean diseases. APS Press, St Paul, pp. 46-48

Guimarães RL, Stolz HU (2005) Oxalate production by Sclerotinia sclerotiorum deregulates guard cells during infection. Plant Physiol 136:3703-3711

Hegedus DD, Rimmer SR (2005) Sclerotinia sclerotiorum: when "to be or not to be" a pathogen? FEMS Microbiol Lett 251:177-184

Lorenz MC, Fink GR (2001) The glyoxylate cycle is required for fungal virulence. Nature 412:83-86

Magro P, Marciano P, Di Lenna P (1984) Oxalic acid production and its role in pathogenesis of Sclerotinia sclerotiorum (sunflower). FEMS Microbiol Lett 24:9-12

Magro P, Marciano P, Lenna PD (1988) Enzymatic oxalate decarboxylation in isolates of Sclerotinia sclerotiorum. FEMS Microbiol Lett 49:49-52

Marciano P, Di Lenna P, Magro P (1983) Oxalic acid, cell wall-degrading enzymes and $\mathrm{pH}$ in pathogenesis and their significance in the virulence of two Sclerotinia sclerotorum isolates on sunflower. Physiol Plant Pathol 22:339-345

Marciano P, Magro P, Favaron F (1989) Sclerotinia sclerotiorum growth and oxalic acid production on selected culture media. FEMS Microbiol Lett 61:57-69CrossRef Maxwell DP (1973) Oxalate formation in Whetzelinia sclerotiorum by oxaloacetate acetylhydrolase. Physiol Plant Pathol 3:279-288

Maxwell DP, Bateman DF (1968a) Influence of carbon source and pH on oxalate accumulation in culture filtrates of Sclerotium rolfsii. Phytopathology 58:13511355

Maxwell DP, Bateman DF (1968b) Oxalic acid biosynthesis by Sclerotium rolfsii. Phytopathology 58:1635-1642

Maxwell DP, Lumsden RD (1970) Oxalic acid production by Sclerotinia sclerotiorum in infected bean and in culture. Phytopathology 60:1395-1398 
Noyes RD, Hancock JG (1981) Role of oxalic acid in the Sclerotinia wilt of sunflower. Physiol Plant Pathol 18:123-132

Pierson P, Rhodes LH (1992) Effect of culture medium on the production of oxalic acid by Sclerotinia trifoliorum. Mycologia 84:467-469

Purdy LH (1979) Sclerotinia sclerotiorum: history, diseases and symptomatology, host range, geographic distribution, and impact. Phytopathology 69:875-880

Rollins JA, Dickman MB (2001) pH signaling in Sclerotinia sclerotiorum: identification of a pacC/RIM1 homolog. Appl Environ Microbiol 67:75-81

Steadman JR (1983) White mold-a serious yield limiting disease of bean. Plant Dis 67:346-350

Vega RR, Corsini D, Le Tourneau D (1970) Nonvolatile organic acids produced by Sclerotinia sclerotiorum in synthetic liquid media. Mycologia 62:332-338

Willetts HJ, Wong JA-L (1980) The biology of Sclerotinia sclerotiorum, S. trifoliorum, and S. minor with emphasis on specific nomenclature. Bot Rev 46:100-165

Wrather JA, Anderson TR, Arsyad DM, Gai J, Ploper LD, Porta-Puglia A, Ram HH, Yorinori JT (1997) Soybean disease loss estimates for the top 10 soybean producing countries in 1994. Plant Dis 81:107-110

Ziman L, Jedryczka M, Šrobárová A (1998) Relationship between morphological and biochemical characteristics of Sclerotinia sclerotiorum isolates and their aggressivity. Z Pflanzenkr Pflanzenschutz 105:283-288

ZirChrom Separations Inc. (2006) Dissociation constants of organic acids and bases. Available at: http://www.zirchrom.com/organic.htm. Cited 11 November 2006 